\title{
High heat flux capabilities of the Magnum-PSI linear plasma device
}

G. De Temmerman ${ }^{a}$, M.A. van den Berg, J. Scholten, A. Lof, H.J. van der Meiden, H.J. van Eck, T.W. Morgan, T.M. de Kruijf, P.A. Zeijlmans van Emmichoven, J.J. Zielinski ${ }^{\mathrm{a}}$

${ }^{a}$ FOM Institute DIFFER, Dutch Institute For Fundamental Energy Research, Association EURATOM-FOM, Trilateral Euregio Cluster, Postbus 120\%, 3430 BE Nieuwegein, The Netherlands

\begin{abstract}
Magnum-PSI is an advanced linear plasma device uniquely capable of producing plasma conditions similar to those expected in the divertor of ITER both steady-state and transients. The machine is designed both for fundamental studies of plasma-surface interactions under high heat and particle fluxes, and as a high-heat flux facility for the tests of plasma-facing components under realistic plasma conditions. To study the effects of transient heat loads on a plasma-facing surface, a novel pulsed plasma source system as well as a high power laser are available. In this article, we will describe the capabilities of Magnum-PSI for high-heat flux tests of plasma-facing materials.
\end{abstract}

Key words: ITER, plasma-surface interactions, ELMs, tungsten, linear plasma device

\section{Introduction}

The divertor plasma in ITER will be characterized by a high density $\left(\geq 10^{21} \mathrm{~m}^{-3}\right)$ and a low electron temperature $\left(\mathrm{T}_{e} \leq 5 \mathrm{eV}\right)$ leading to high heat $\left(\geq 10 \mathrm{MW} \cdot \mathrm{m}^{-2}\right)$ and particle fluxes (up to $10^{24} \mathrm{~m}^{-2} \mathrm{~s}^{-1}$ or $1.6 \times 10^{5} \mathrm{~A} \cdot \mathrm{m}^{-2}$ ) [1]. Assuming ion acceleration in the electrostatic sheath, the ions will have energies below $50 \mathrm{eV}$, at the divertor strike-point. In addition, the very high transient localized heat fluxes caused by so-called Edge Localized Modes (several $\mathrm{GW} \cdot \mathrm{m}^{-2}$ for $0.5-1$ $\mathrm{ms}$ ) is high enough to lead to material erosion, melting and vaporization for most materials, and represent a serious concern for the lifetime of the plasma-facing components.

The understanding and control of plasma-wall interactions is of paramount importance for the successful deployment of nuclear fusion energy and rely on facilities able to reproduce the expected plasma conditions. However, with the exception of the Alcator C-Mod tokamak [2], neither ex-

Email addresses: g.c.detemmerman@differ.nl (G. De Temmerman) isting tokamaks nor laboratory devices were capable of mimicking closely enough the conditions expected in future fusion reactors. Linear plasma generators, such as the PISCES facilities at UC San Diego $[3,4]$ or the NAGDIS facilities at the University of Nagoya [5], have long been used for the study of plasma-surface interactions under fusion-relevant conditions. The achievable ion flux density in those devices is typically limited to $1 \times 10^{23} \mathrm{~m}^{-2} s^{-1}$ in steady-state, a factor of 10 lower than what is expected in the divertor of ITER. In addition, it is currently not possible to generate very short $(\sim \mathrm{ms})$ high power density transient plasmas. Instead, high power lasers are used to combine a plasma environment and transient heat fluxes and get some insights into the effects of ELMs on plasma-exposed surface $[6,7]$, albeit missing the transient particle flux associated with an ELM. Powerful plasma guns [8] can be used to study the effect of powerful transient plasma pulses on surfaces, albeit in the absence of continuous plasma loading. Filling the gap between those existing devices and future reactors lead to the development of the Magnum-PSI linear plasma generator $[9,10]$ which began op- 
erations in the beginning of 2012 [11] and provides for the first time the combination of a high flux steady-state plasma and frequent ELM-like plasma pulses. An overview of the current status of Magnum-PSI can be found in [11]. In this contribution, we will focus on the description of the high-heat flux capabilities of the device.

\section{Scientific purpose and design criteria of Magnum-PSI}

\subsection{The strongly-coupled regime}

Magnum-PSI has been designed to study plasmasurface interactions under the so-called 'stronglycoupled' regime which is reached when the mean free-path of the particles released from the surface, via reflection or erosion, is smaller than the plasma size so that they are trapped in the plasmasurface interaction region. In the case of the chemical sputtering of graphite by hydrogen plasmas, for example, for $0.3 \mathrm{eV} \leq \mathrm{T}_{e} \leq 2 \mathrm{eV}$, the ionization of the hydrocarbons released from the surface primarily occurs through charge exchange. The ionization mean-free path strongly depends on the plasma density in front of the surface and for instance decreases from $3.2 \mathrm{~mm}$ for $\mathrm{n}_{e}=1 \times 10^{20} \mathrm{~m}^{-3}$ to $0.8 \mathrm{~mm}$ for $\mathrm{n}_{e}=4 \times 10^{20} \mathrm{~m}^{-3}$. This has to be compared with the Full-Width at Half Maximum of the plasma beam which is about $25 \mathrm{~mm}$ in Magnum-PSI. As a consequence, every eroded particle will experience a cycle of erosion/re-deposition events before it can eventually escape the plasma beam. Modeling shows that every $\mathrm{CH}$ molecule eroded from the surface, for a density of $4 \times 10^{20} \mathrm{~m}^{-3}$, will visit the surface in average 19 times before actually escaping the plasma beam [12]. This gives rise to strong re-organization of carbon surfaces with the quick growth of large carbon particles which are re-deposited on the surface [13].

In addition, the particle flux to the surface is so high that every surface atom is visited by reactive particles from the plasma with a frequency higher than the inverse local surface relaxation time, driving the surface into states far from equilibrium. Low-energy ions $(\leq 10 \mathrm{eV})$, having kinetic energies in the range of the interatomic binding energies, can transfer their energies very effi- ciently to surface atoms thus enhancing adatoms mobility, leading to enhanced surface diffusion and reactivity for example. Those conditions promote self-organization effects and the appearance of novel structures, an overview of which is presented in [14].

\subsection{Design criteria}

Magnum-PSI was designed to generate plasma conditions similar to those expected in the divertor of ITER, the main design criteria can be summarized as follows:

- Divertor relevant plasma conditions, i.e. a plasma with high density and low temperature with hydrogen/deuterium as a process gas. The choice was made to use a highpressure plasma source, the so-called cascaded arc source [15], which typically operates at pressures around $10^{4} \mathrm{~Pa}$, which is to be compared with the pressure of a few $\mathrm{Pa}$ in typical $\mathrm{LaB}_{6}$ assisted arc discharges [3].

- Neutral pressure around the target determined almost entirely by the recycling at the target, i.e. that the influx of cold neutrals from the source should be negligible in front of the ion flux. This requires the use of differential pumping which has to be optimized for the high gas flows required for the plasma generation. More details about the design of the differential pumping can be found in [16].

- A high magnetic field: the machine has been designed around a $3 \mathrm{~T}$ superconducting magnet $[17]$

- A realistic target geometry allowing large targets to be exposed at a shallow angle with respect to the magnetic field (2.9 degrees) [18].

- In-vacuo surface analysis capabilities.

\subsection{Current status}

Delays in the delivery of the superconducting have lead to temporary alterations of the machine design to allow for the installation of oil-cooled copper magnets [11] generating a magnetic field of up to $1.73 \mathrm{~T}$ close to the plasma source. A general view of the machine fitted with copper coils is shown in fig. 1. Those magnets are not able 

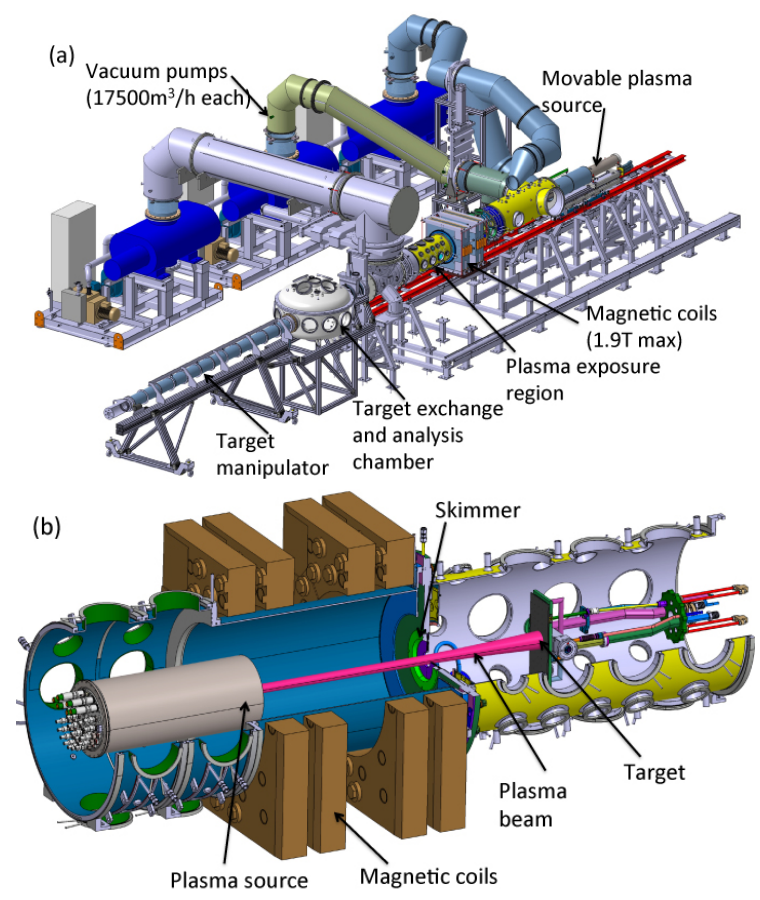

Figure 1: Schematic overview of the Magnum-PSI linear plasma device. (a) Global view of the device with a description of the main components, (b) cut-out view through the vacuum vessel illustrating the geometry of the magnetic coil arrangement as well as a typical plasma exposure.

to operate in steady-state at high fields and, similarly to Pilot-PSI, Magnum-PSI currently operates in a pulsed mode with the discharge duration varying between $6 \mathrm{~s}$ and $112 \mathrm{~s}$ for magnetic fields of $1.73 \mathrm{~T}$ and $0.43 \mathrm{~T}$ respectively. In addition, a 2-stage differential pumping system is currently in use (instead of the originally designed 3-stage) with the source chamber and the target chamber being separated by a conically-shaped skimmer. Finally, the target is now installed in a diverging magnetic field. With this configuration, plasma densities of up to $7 \times 10^{20} \mathrm{~m}^{-3}$ and temperatures of up to $3.5 \mathrm{eV}$ were achieved in hydrogen [11], with very high ionization efficiencies of up to $63 \%$ [19].

\section{High-heat flux capabilities}

\subsection{Target system}

The target manipulator has been built to allow a large flexibility in terms of sample shape and size to be exposed. Three different target holders are available and can be exchanged relatively easily during a maintenance day [18]. A multitarget holder allows 5 disc-shaped samples to be installed and sequentially exposed to the plasma beam. The samples are clamped onto a watercooled copper support using a tantalum clamping ring, while a thin sheet of Grafoil is used to improve the thermal contact. A generic rotatable holder consisting of a stainless steel plate of $320 \times 120 \times 10 \mathrm{~mm}$ with 12 cooling channels milled into it, onto which a $2.5 \mathrm{~mm}$ copper lid is brazed is also available. 52 holes, $24 \mathrm{~mm}$ apart in length and width, can be used for sample mounting and insertion of thermocouples for example.

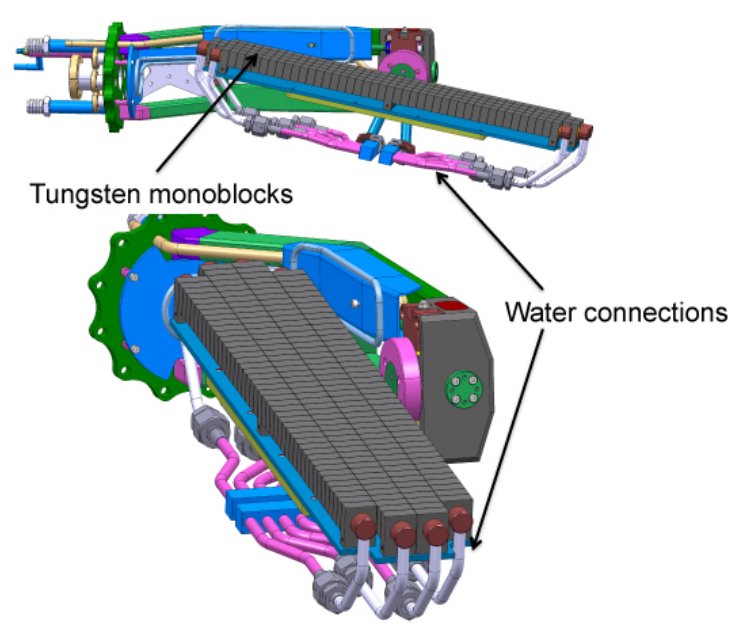

Figure 2: Target holder system with an ITER-like actively cooled tungsten target made of tungsten monoblocks. The target holder can accomodate samples with dimensions up to $600 \times 120 \mathrm{~mm}$. In such a configuration, plasma exposure is possible with very shallow angles $\left(2.9^{\circ}\right)$ between the magnetic field and the surface.

Finally, a manipulator has been developed to allow the exposure of targets with a wide range of sizes and shapes. The maximum target dimensions are $600 \times 120 \mathrm{~mm}$, for a weight of $100 \mathrm{~kg}$. Fig. 2 illustrates the holder equipped with an ITER-like target consisting of tungsten monoblocks. With such a configuration, an inclination angle of $2.9^{\circ}$ is possible. 


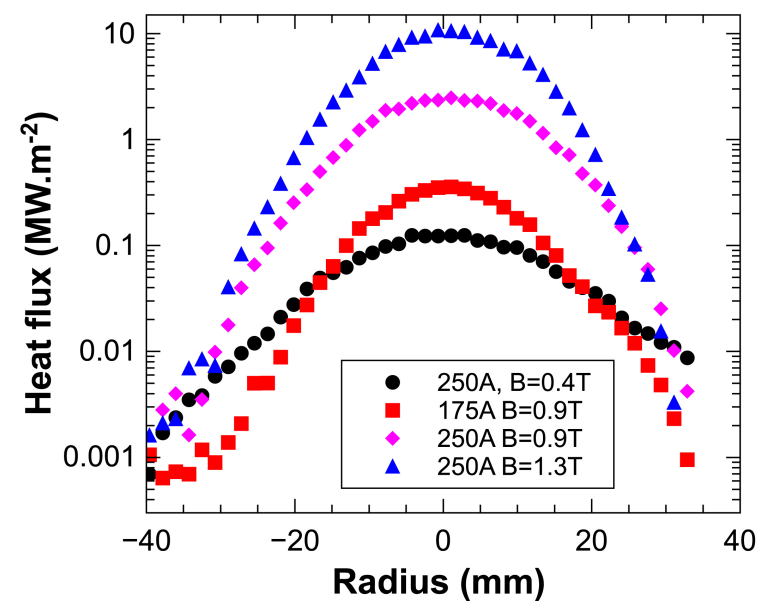

Figure 3: Steady-state heat flux profiles (determined from plasma parameters) evolution as a function of discharge current and magnetic field strength.

\subsection{Steady-state heat loads}

The heat loads to a plasma-exposed target can be determined by different diagnostics, including calorimetry, infrared thermography and thomson scattering. For the latter, the plasma parameters (density, temperature) are determined $25 \mathrm{~mm}$ away from the surface with a spatial resolution of $1.6 \mathrm{~mm}$ [20]. The ion flux to the surface is then estimated by assuming that ions are accelerated over the pre-sheath up to the sound speed. The power flux to the target can be derived from the ion flux using the sheath heat transmission factor [21], which has been experimentally determined for the plasma conditions of interest here in [22]. The power flux profile is shown in fig. 3 as a function of discharge current (i.e. input power to the plasma source) and magnetic field at the source location, for a hydrogen gas flow of $5 \mathrm{slm}$ $\left(1 \mathrm{slm}=4.4 \times 10^{20} \mathrm{H}_{2} \cdot s^{-1}\right)$. As seen in fig. 3, the target heat flux is strongly dependent on both the discharge current and magnetic field, and peak heat fluxes of about $12 \mathrm{MW} \cdot \mathrm{m}^{-2}$ are reached for a source current of $250 \mathrm{~A}$ and a magnetic field of $1.4 \mathrm{~T}$. It should be noted that the maximum achievable power density in Magnum-PSI is currently limited by the magnetic geometry as the magnetic field strength decreases by a factor 5 from source to target reducing the plasma density by broadening the plasma beam. Heat fluxes up to $50 \mathrm{MW} \cdot \mathrm{m}^{-2}$ were achieved in Pilot-PSI [23] with a straight field of $1.6 \mathrm{~T}$, and it is expected that similar heat flux levels will be possible in Magnum-PSI after the installation of the superconducting magnet system.

\subsection{Transient heat loads}
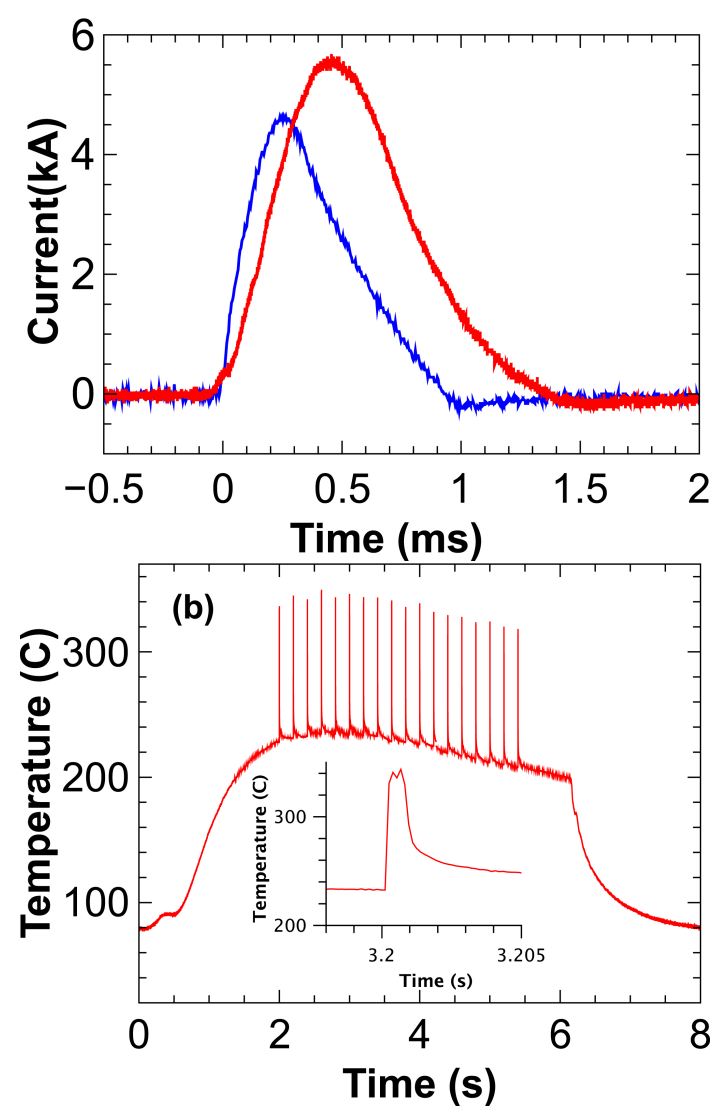

Figure 4: (a) Temporal evolution of the discharge current in the plasma source for different configurations of the pulsed power supply. In one case, all capacitors were triggered simultaneously, while in the second case a delay of $50 \mu$ s was introduced between the capacitors thus changing the current risetime. (b) Temporal evolution of the peak surface temperature of a tungsten surface during combined steady-state/transient plasma exposure. The steady-state discharge duration was $6 \mathrm{~s}$, and the transients started at $\mathrm{t}=2 \mathrm{~s}$ for a duration of $3 \mathrm{~s}$ with a repetition rate of $5 \mathrm{~Hz}$. The inset of (b) shows the temperature evolution during a single ELM-like event.

A unique pulsed plasma source system has been developed on Pilot-PSI to allow the superimposition of a de plasma and a high-power plasma impulse with a single plasma source [24]. The transient heat loads are generated by transiently increasing the input power in the plasma source, 
which is achieved by a capacitor bank system coupled in parallel with the DC power supply. The pulse duration is about $1.3 \mathrm{~ms}$ and heat fluxes in excess of $1 \mathrm{GW} \cdot \mathrm{m}^{-2}$ have been achieved in hydrogen, helium and argon. The system is aimed at studying the possibility of synergistic effects caused by the simultaneous exposure of a metallic surface to a divertor relevant high flux plasma and a transient heat/particle source [25].

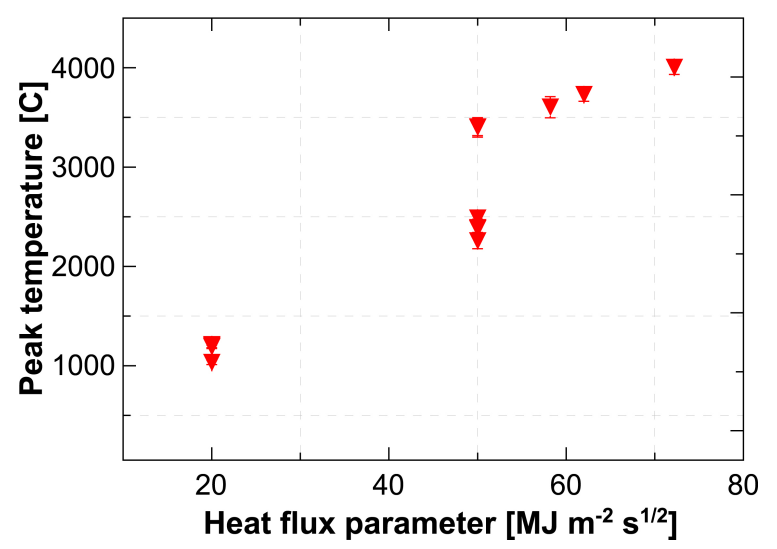

Figure 5: Evolution of the peak surface temperature, measured using a fast infrared camera, of a tungsten surface as a function of the laser induced heat flux parameter.

On Pilot-PSI, the repetition rate is limited to one pulse per discharge because of the charging time of the capacitors. A high-repetition rate system has been designed and commissioned for Magnum-PSI, allowing a $10 \mathrm{~Hz}$ repetition rate for peak heat fluxes of $1 \mathrm{GW} \cdot \mathrm{m}^{-2}$. The system is based on 28 individual charging/discharging units which can be independently triggered allowing the shape of the pulse to be tailored to the needs. Fig. 4a shows two different waveforms of the discharge current obtained with different delay times between the capacitors. In one case, the capacitors were triggered simultaneously resulting in a current risetime of about $250 \mu \mathrm{s}$. In the second case, a delay of $50 \mu$ s was introduced between the capacitors resulting in a risetime of about $500 \mu \mathrm{s}$. More details about the ELM simulation system will be published elsewhere.

Commissioning of the system on Magnum-PSI has been completed, fig. 4b shows the temporal evolution of the peak surface temperature of a tungsten target exposed to simultaneous steady- state/transients plasma conditions. The plasma duration was set to $6 \mathrm{~s}$, and the ELM-like pulses were started at $\mathrm{t}=2 \mathrm{~s}$ and stopped at $\mathrm{t}=5 \mathrm{~s}$. The repetition rate was $5 \mathrm{~Hz}$. As seen, the plasma pulses do not disturb the steady-state plasma loading, and the surface temperature returns to its steady value quickly after the pulse, the resulting time trace ressembles very closely what is observed in tokamaks. The inset in fig. $4 \mathrm{~b}$ shows the surface temperature evolution during a single ELM-like event. The framerate of the infared camera $(7.5 \mathrm{kHz})$ is high enough to capture the dynamics of the surface temperature evolution. The infrared camera is calibrated against a spectal pyrometer to determine the surface emissivity, and the heat flux calculated from the surface temperature measurements are in reasonable agreement with the heat flux derived from thomson scattering- the detailed comparison will be published elsewhere. The slight decrease in the interELM surface temperature is caused by the decrease of magnetic field over time. Here also, the peak power density during plasma-induced transients is currently limited by the magnetic field geometry to values of about $200 \mathrm{MW} \cdot \mathrm{m}^{-2}$.

In addition to the pulsed plasma system, a high power laser (LASAG FLS N, $1064 \mathrm{~nm}$ ) is coupled to the target chamber for pulsed heating of the surface during plasma exposure. The pulse duration can be tuned between 0.5-5 ms, the maximum laser energy is $60 \mathrm{~J}$. The spot size is about $3 \mathrm{~mm}$, which translates to a maximum heat flux parameter of $75 \mathrm{MJ} \cdot \mathrm{m}^{-2} \cdot \mathrm{s}^{1 / 2}$, corresponding to a peak surface temperature for a tungsten surface of $4000^{\circ} \mathrm{C}$ i.e. above the melting point of tungsten (fig.5)

Fig. 6 shows the evolution of the surface morphology of a tungsten target exposed to simultaneous steady-state hydrogen plasma and pulsed laser irradiation in Magnum-PSI. The magnetic field was triggered for a duration of $10 \mathrm{~s}(\mathrm{~B}=1.4 \mathrm{~T})$, the sample was biased to $-30 \mathrm{~V}$ and the base temperature was $200^{\circ} \mathrm{C}$. The laser repetition rate was set to $10 \mathrm{~Hz}$ and the same procedure was repeated 10 times to accumulate a total of 1000 laser pulses. Two different heat flux parameters were used: $20 \mathrm{MJ} \cdot \mathrm{m}^{-2} \cdot \mathrm{s}^{1 / 2}$ and $40 \mathrm{MJ} \cdot \mathrm{m}^{-2} \cdot \mathrm{s}^{1 / 2}$, respec- 


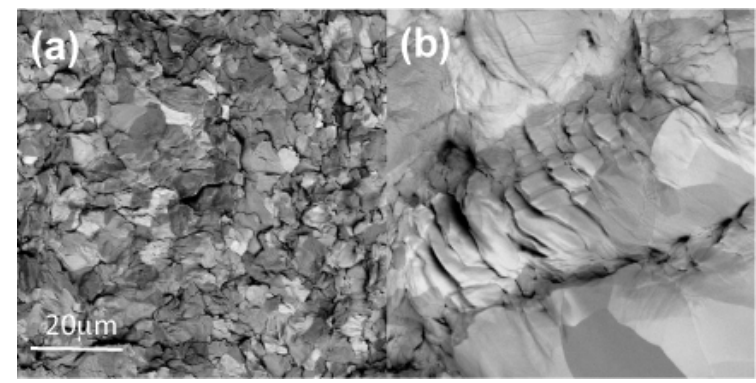

Figure 6: Scanning electron microscopy images of tungsten samples after exposure to $100 \mathrm{~s}$ of hydrogen plasma at $200^{\circ} \mathrm{C}$, together with 1000 laser pulses with heat flux parameters (a) $20 \mathrm{MJ} \cdot \mathrm{m}^{-2} \cdot \mathrm{s}^{1 / 2}$ and (b) $40 \mathrm{MJ} \cdot \mathrm{m}^{-2} \cdot \mathrm{s}^{1 / 2}$.

tively. For the lowest laser energy, surface roughening and the development of cracks at grain boundaries can clearly be seen. It should be mentioned that under otherwise similar laser loading conditions, no surface modifications can be observed in the absence of plasma exposure [26], outlining the possible strong role of hydrogen plasma exposure on the surface damage of tungsten as well as the importance of synergistic effects arising during simultaneous plasma/transients exposure [25]. Further studies will be carried out to compare the damage induced by laser and pulsed plasma heating, respectively, and elucidate the role of the increased particle flux during a plasma pulse on the surface response.

\section{Summary}

Magnum-PSI is designed both for fundamental studies of plasma-surface interactions under extreme heat and particle fluxes, and as a high-heat flux facility for the tests of plasma-facing components under realistic plasma conditions. In this paper, we have described the capabilities of the device for steady-state and transient heat loading. Although the machine is currently limited in pulse duration and maximum power density by the use of conventional copper coils, heat loads in excess of $10 \mathrm{MW} \cdot \mathrm{m}^{-2}$ can be produced routinely in quasi steady-state. In addition, a unique ELM-simulation system has been developed to superimpose powerful ELM-like plasma pulses to the steady-state plasma with a repetition rate of $10 \mathrm{~Hz}$. The peak power density during the plasma pulses is about $200 \mathrm{MW} \cdot \mathrm{m}^{-2}$ and only limited by the current magnetic field geometry. This system is complemented by a high power laser system for transient heating of the plasma-exposed surface. This provides the opportunity to compare the damages induced by a pulsed plasma to the pure heating induced by the laser. Together with the flexible target system, this makes MagnumPSI a unique facility to study the power handling of candidate plasma-facing materials.

\section{References}

[1] R.A. Pitts, et al, J. Nucl. Mater., 415 (2011) S957

[2] B. Lipschultz, et al, Phys. Plasmas, 6 (1999) 1907

[3] D. M. Goebel, G. Campbell and R. W. Conn, J. Nucl. Mater., 121, (1984) 277

[4] Y. Hirooka et al., J. Vac. Sci. Technol. A, (1990) 8

[5] N. Ohno, et al, Nucl. Fusion, 41 (2001) 1055

[6] S. Kajita, N. Ohno, S. Takamura, W. Sakaguchi and D. Nishijima, Appl. Phys. Lett. 91 (2007) 261501

[7] K.R. Umstadter, R. Doerner and G. Tynan, Phys. Scr. T138 (2009) 014047

[8] I.E. Garkusha, et al, J. Nucl. Mater., 390-391 (2009) 814-817

[9] B. de Groot, et al, Fus. Eng. Des., 66-68 (2003) 413

[10] A.W. Kleyn, N.J. Lopes Cardozo, and U. Samm, Phys. Chem. Chem. Physics, 8 (2006) 1761

[11] J. Scholten et al, these proceedings

[12] G.A. van Swaaij, et al, J. Nucl. Mater., submitted

[13] K. Bystrov, L. van der Vegt, G. De Temmerman, C. Arnas, and L. Marot, J. Vac. Sci. Technol. A, in press

[14] G. De Temmerman et al, Acta Polit., in press

[15] M.C.M. van de Sanden, et al, Rev. Sci. Instrum., 63 (1992) 3369

[16] H.J.N. van Eck, et al, Plasma Sources. Sci. Technol., 20 (2011) 045016

[17] H.J.N. van Eck, et al, Fus. Eng. Des., 82 (2007) 1878

[18] M.A. van den Berg, et al, Fus. Eng. Des., 86 (2011) 1745

[19] H.J.N. van Eck, Appl. Phys. Lett., in press

[20] H.J. van der Meiden, et al, Rev. Sci. Instrum., in press

[21] P.C. Stangeby, The plasma boundary of Magnetic Fusion Devices, Taylor and Francis Group, London 2000

[22] M.A. van den Berg, et al, J. Nucl. Mater., submitted

[23] G. De Temmerman, et al, Nucl. Fusion, in press

[24] J.J. Zielinski, H J van der Meiden, T W Morgan, D C Schram and G De Temmerman, Plasma Sources Sci. Technol., 21 (2012) 065003

[25] G. De Temmerman, et al, Nucl. Fusion, 51 (2011) 073008

[26] T.W. Morgan , J.J. Zielinski, B.J. Hensen, H.Y. Xu, L. Marot, and G. De Temmerman, J. Nucl. Mater., in press 